\title{
CHAPITRE PRÉLIMINAIRE
}

\section{LE DROIT PUBLIC ALLEMAND DEPUIS LES TRAITÉS DE WESTPHALIE JUSQU'À LA FIN DU XVII ${ }^{e}$ SIÈCLE}

\section{Le Saint-Empire, ses ordres et ses institutions}

L'Empire, à compter du couronnement de Charlemagne, le jour de Noël de l'an 800 , jusqu'à sa dissolution, en 1806 , eut un peu plus de mille ans d'existencel. À partir de la rénovation impériale de $962^{2}$, il perdura presque neuf cents ans. Abstraction faite de la question de savoir si le Saint-Empire fut un >État< plutôt archaïque ou au contraire moderne, cette longévité est un signe indéniable de sa réussite. Si la théorie de la translation de l'empire faisait de l'Allemagne le principal dépositaire de l'héritage de l'ancien Empire romain, l'histoire du SaintEmpire ne se limitait pas à l'Allemagne; tout au long des époques médiévale et moderne, l'Empire conservait certains droits en Italie. Pour cette raison, le droit féodal lombard faisait partie du droit public du Saint-Empire.

La sainteté et la romanité se reflétaient dans la dénomination du Saint Empire romain de la nation allemande. Ses structures étaient si singulières que même les contemporains, à l' instar du juriste allemand Samuel Pufendorf, le considéraient comme une sorte de monstre. Pourtant, cet empire jouissait d'un prestige énorme, non seulement chez les Allemands, mais aussi dans les pays voisins, et notamment en France. Même si, au XVIII' siècle, Voltaire, comme on le sait bien, jugea que cet empire n'était ni saint, ni romain, ni empire, nous allons voir qu'il ne porta point de jugement exclusivement négatif sur ce soi-disant »empire«. Pour cette raison, on peut estimer que la singularité du Saint-Empire gêna plus ses historiographes que ses contemporains.

1 Il n'est pas dans notre objectif de donner, dans ce chapitre, une bibliographie exhaustive des travaux qui existent sur l'histoire constitutionnelle du Saint-Empire. Les contributions concernant des aspects particuliers de cette Constitution seront évoquées dans les différents chapitres de notre livre. Nous voudrions seulement signaler quelques ouvrages fondamentaux, surtout les publications en langue française. Sur le problème en général, on peut consulter NoËL, Saint-Empire. Pour l'Empire médiéval, on pourra se rapporter à Francis RAPP, Le Saint Empire romain germanique. D'Otton le Grand à Charles Quint, [Paris] 22003 (Points Histoire, H328). La meilleure histoire de la Constitution de l'Empire à l'époque moderne, car brève et précise, est celle de Heinz DuchHARDT, Deutsche Verfassungsgeschichte 1495-1806, Stuttgart, Berlin, Cologne 1991 (Urban-Taschenbücher, 417). Pour l'histoire du droit public d'Allemagne aux XVII ${ }^{\mathrm{e}}$ et XVIII ${ }^{\mathrm{e}}$ siècles, on dispose maintenant de la version française de l'ouvrage classique bien que relativement récent de STOLleIS, Histoire du droit public. Signalons aussi un ouvrage sur l'histoire de l'administration, qui peut servir à approfondir le sujet, avec la bonne bibliographie qu'il comporte: Kurt G. A. JESERICH, Hans POHL, Georg-Christoph voN UNRUH (dir.), Deutsche Verwaltungsgeschichte, vol. I: Vom Spätmittelalter bis zum Ende des Reiches, Stuttgart 1983.

${ }^{2}$ Le 2 février 962, Otton Ir fut couronné empereur en la basilique Saint-Pierre de Rome. 
$\mathrm{Au}$ lieu de conserver partout ses traditions médiévales, le Saint-Empire sortit profondément transformé de la période réformatrice qu'il parcourut de 1495 (diète de Worms) à 1555 (paix de religion d'Augsbourg). La réforme des institutions impériales, pour laquelle la langue allemande inventa un seul mot composé (Reichsreform $)^{3}$, avait déjà été au cœur du débat politique au XVe siècle. Le théologien et cardinal allemand Nicolas de Cues l'avait évoquée dans sa »Concordantia catholica«, en 1432, de même qu'un traité anonyme datant du milieu du siècle est connu comme la »Réforme de l'empereur Sigismond«. Cette réforme institutionnelle, au lieu de consacrer à tous les égards la participation des ordres, contribua au renforcement du pouvoir de l'empereur et des institutions impériales, de sorte qu'il est également faux de parler d'un déclin continuel de l'Empire que d'un Empire incapable de s'adapter aux évolutions historiques qui ont caractérisé l'Europe depuis la Renaissance. Certes, le parti de la réforme parmi les états de l'Empire - que l'archevêque de Mayence, Berthold von Henneberg, conduisit à l'époque de Maximilien Ier - avait pour objectif d'affirmer l'autonomie des États territoriaux ${ }^{4}$ plutôt que le pouvoir de l'empereur; mais au lieu d'insister sur l'antagonisme qui aurait existé entre ce dernier et les ordres, il convient de prendre acte de la collaboration féconde entre tous les pouvoirs qui caractérisa l'activité réformatrice à cette période où les diètes jouèrent un rôle primordial.

Cela ne veut pas dire que le Saint-Empire fut exempt de conflits aux Temps modernes. Au contraire, la Réforme de 1517, qui prit une dimension constitutionnelle à partir de 1521, lorsque Charles Quint mit Luther au ban de l'Empire, à la diète de Worms, entraîna de nombreux conflits politiques et militaires, qui ne s'achevèrent qu'en 1648. Cependant, à part la Bulle d'or de 1356 qui avait réglé l'élection impériale et la partie du droit public concernant les électeurs et la transition des pouvoirs à chaque fin de règne, et à part les capitulations impériales de Léopold Ier et de ses successeurs, toutes les lois fondamentales du Saint-Empire datent de la période qui va de 1495 à 1648; l'activité législative des empereurs et des ordres témoigne du bon fonctionnement des institutions (sans que nous cherchions à minimiser leurs dysfonctionnements, notamment à la veille de la guerre de Trente Ans), et de leur capacité de s'adapter à de nouvelles donnes politiques et religieuses.

3 C'est notamment Heinz ANGERMEIER qui forgea ce concept, cf. ID., Die Reichsreform 1410-1555. Die Staatsproblematik in Deutschland zwischen Mittelalter und Gegenwart, Munich 1984.

4 Par le terme "états de l'Empire«, nous désignons les princes électeurs, princes et villes de l'Empire représentés à la diète générale (les ordres; Reichsstände). Souvent les Français les appelaient simplement les »princes«. Certaines principautés constituaient un territoire avec les caractéristiques d'un véritable État au XVIfe et surtout au XVIII ${ }^{e}$ siècle (environ cinquante sur plus de trois cents princes et villes). Toutefois, elles continuaient à faire partie du Saint Empire romain germanique. L'allemand a développé à cette fin le concept de Territorialstaat, que l'on peut traduire en français par »État territorial «. Par contre, à l'exemple des Français de l'époque moderne, nous employons le terme d'»états provinciaux « à l'égard des ordres relevant d'un territoire (Landstände). 
Durant toute l'époque moderne, la Bulle d'or continua à régler les formalités de l'élection à l'empire, au nombre des électeurs près, qui fut porté de sept à huit par la création de l'électorat bavarois, en 1623 et 1648, et de huit à neuf par l'admission de Brunswick-Lunebourg (Hanovre) au collège électoral, en 1692 et $1708^{5}$. À partir de 1508 , l'empereur porta ce titre dès son avènement. Le titre de roi des Romains fut dorénavant appliqué à son successeur, souvent élu du vivant de l'empereur régnant (le terme technique désignant cette forme d'élection est vivente Imperatore). Malgré cette particularité qui contribua à assurer la continuité dynastique au profit de la maison d'Autriche jusqu'en 1740 et, à une exception près (1742-1745), jusqu'à la fin du Saint-Empire, il y eut bien des interrègnes. Durant la vacance du trône impérial, trois princes de l'Empire étaient appelés par la Bulle d'or à assurer l'exercice du pouvoir impérial: l'électeur de Saxe dans les pays de droit saxon, les électeurs de la maison de Wittelsbach (Bavière ou Palatinat) pour les pays de droit rhénan et le duc de Savoie en Italie ${ }^{6}$. Depuis le dernier couronnement d'un empereur en Italie - qui fut celui de Charles Quint, à Bologne, en 1530 - les empereurs se faisaient couronner en général peu après leur élection par les électeurs du Saint-Empire, à Francfort, non plus par le pape, mais par l'électeur de Mayence ou par l'archevêque du ressort du lieu du couronnement.

Les capitulations impériales que les empereurs et les électeurs avaient coutume de négocier avec le futur souverain depuis 1519 fixaient les conditions de l'exercice du pouvoir impérial, mais elles n'étaient pas toujours prises au pied de la lettre par les empereurs une fois qu'ils avaient accédé à l'empire. Malgré les tentatives des autres ordres d'être admis à la négociation de la capitulation et à l'établissement d'une capitulation perpétuelle, depuis 1648, la rédaction des capitulations resta entre les mains des électeurs, et le projet de capitulation perpétuelle n'aboutit jamais.

L'exercice de certains droits était réservé à l'empereur; ces iura reservata étaient de trois sortes: droits sacrés (iura sacra), découlant de son titre d'advocatus Ecclesiae et comprenant notamment la collation de certains bénéfices; droits de grâce (iura gratialia), tels que le pouvoir de créer des nobles et celui d'accorder des privilèges; droits féodaux (iura feudalia), qui lui revenaient en tant que suzerain et dont l'investiture des fiefs immédiats de l'Empire faisait partie. Or, la législation et la décision de la guerre et de la paix constituaient les iura comitilia que l'empereur était obligé d'exercer de concert avec les ordres réunis à la diète ${ }^{7}$.

Mais l'empereur n'était point le seul pouvoir central de l'Empire: l'archevêque de Mayence en tant qu'archichancelier (Reichserzkanzler) avait une certaine influence sur la conduite technique des affaires et nommait le vice-chancelier qui

5 Jusqu'en 1756, ce nombre ne fut plus modifié; nous passons sous silence les changements ultérieurs, depuis l'extinction de la Bavière, en 1777, jusqu'en 1806.

${ }^{6} \mathrm{Ce}$ dernier titre était pourtant plutôt honorifique que réel à l'époque moderne.

7 Certains droits, qui diminuèrent à partir de 1648 , devaient être exercés par l'empereur et les électeurs. 
dirigeait la Chancellerie impériale aulique (Reichshofkanzlei) de Vienne ${ }^{8}$, chargée d'établir les documents impériaux et divisée en une »expédition" allemande et une »expédition « latine, qui était le reflet de la romanité de l'Empire.

Comme résultat d'une distinction plus nette entre les organes administratifs patrimoniaux et impériaux, le Conseil aulique (Reichshofrat) se concentra dès le milieu du XVII ${ }^{e}$ siècle exclusivement sur ses tâches impériales, qui étaient de trois sortes: il était surtout une suprême instance judiciaire mais en même temps un conseil politique et une chambre féodale. Le second tribunal souverain de l'Empire était la Chambre impériale (Reichskammergericht).

Le rôle de législateur était essentiellement assuré par la diète: votés par les ordres, les projets de lois avaient besoin de la ratification par l'empereur et de la promulgation pour devenir des recès, c'est-à-dire pour acquérir force de loi ${ }^{9}$. L'exécution des recès de la diète entrait dans les compétences des dix cercles créés par Maximilien $\mathrm{I}^{\mathrm{er}}$, entre 1500 et 1512 , comme unités régionales intermédiaires entre l'Empire et les centaines de principautés et de villes qui le composaient. Ces cercles (Reichskreise) étaient le cercle de la Rhénanie électorale (Kurrheinischer Kreis), du Haut-Rhin (Oberrheinischer Kreis), du Bas-Rhin Westphalie (Niederrheinisch-Westfälischer Kreis), de Bourgogne (Burgundischer Kreis), de BasseSaxe (Niedersächsischer Kreis), de Haute-Saxe (Obersächischer Kreis), de Souabe (Schwäbischer Kreis), de Franconie (Fränkischer Kreis), de Bavière (Bayerischer Kreis) et d'Autriche (Österreichischer Kreis) ${ }^{10}$.

La noblesse immédiate était exclue de cette répartition mais formait trois cercles équestres (Rhénanie, Souabe et Franconie). La couronne de Bohême et les pays qui en relevaient (Lusace, Silésie) ne faisaient pas non plus partie des cercles, puisque la Bohême, tout en faisant de iure partie du Saint-Empire, ne parti-

${ }^{8}$ La création de la chancellerie aulique autrichienne sépara plus clairement, à partir de 1620 , l'administration des pays héréditaires et les affaires impériales.

${ }^{9}$ Le terme »recès« dérive du mot latin désignant le retrait des ordres (recesserunt) à la fin de la diète qui de iure, ne fut jamais permanente, mais le fut de facto à partir de 1663; étant donné l'importance de la diète pour la vision française du Saint-Empire, nous reviendrons sur sa composition et son fonctionnement à la fin de ce chapitre.

${ }^{10}$ Sur les cercles de l'Empire, qui ont fait l'objet d'un certain nombre de travaux très importants ces dernières décennies, voir notamment Peter Claus HaRTManN, Der Bayerische Reichskreis (1500-1803). Strukturen, Geschichte und Bedeutung im Rahmen der Kreisverfassung und der allgemeinen institutionellen Entwicklung des Heiligen Römischen Reiches, Berlin 1997 (Schriften zur Verfassungsgeschichte, 52); dans la première partie, Hartmann traite de l'histoire des cercles de l'Empire en général; Bernhard SICKEN, Der Fränkische Reichskreis im Zeitalter der Aufklärung, dans: Peter Claus HaRTMANN (dir.), Regionen in der Frühen Neuzeit, Berlin 1994 (Zeitschrift für Historische Forschung, cahier suppl. 17), p. 61-77; Winfried DotZaUER, Die deutschen Reichskreise in der Verfassung des Alten Reiches und ihr Eigenleben (1500-1806). Darmstadt 1989; ID., Die deutschen Reichskreise (1383-1806). Geschichte und Aktenedition, Stuttgart 1998; Heinz MoHNHAUPT, Die verfassungsrechtliche Einordnung der Reichskreise in die Reichsorganisation, dans: Karl Otmar Freiherr vON ARETIN (dir.), Der Kurfürst von Mainz und die Kreisassoziationen 1648-1746, Wiesbaden 1975 (Veröffentlichungen des Instituts für Europäische Geschichte Mainz, cahier suppl. 2), p. 1-30. Nous indiquerons d'autres éléments de bibliographie dans les chapitres suivants. 
cipait guère (surtout pas avant 1708) à ses institutions ${ }^{11}$. En revanche, si la soi-disant plénipotence impériale servait de relais administratif en Italie, il ne faut pas oublier que la Savoie appartenait juridiquement au cercle du Haut-Rhin ${ }^{12}$.

Ces cercles étaient dotés d'un certain nombre d'institutions: dans ce contexte, il faut notamment évoquer les diètes circulaires, que les Français nommaient aussi »diétines« (Kreistage) et auxquelles ils s'intéressaient surtout dans la deuxième moitié du XVII siècle, y envoyant leurs émissaires ou leurs agents ${ }^{13}$. L'exécutif était confié aux princes convocateurs (Kreisausschreibende Fürsten) qui étaient en général les princes séculiers et ecclésiastiques les plus puissants de leurs cercles respectifs. À part cela, les cercles disposaient de leur propre chancellerie, d'un trésor, d'une milice circulaire, etc.

Leur fonction était triple: premièrement, les cercles jouaient le rôle de relais administratifs de l'Empire, chargés d'exécuter ses lois, les jugements de ses tribunaux souverains, etc.; deuxièmement, ils contribuaient à l'organisation de sa défense, et c'était en particulier dans cette fonction militaire qu'ils retenaient l'intérêt des Français; troisièmement, les cercles devaient servir à coordonner les solutions apportées aux problèmes d'intérêt régional (monétaires, économiques, etc.). Cette dernière fonction était sans importance majeure dans les cercles qui se composaient essentiellement d'un seul territoire ou d'un nombre assez restreint d'États territoriaux (Autriche, Bavière) mais elle s'avérait indispensable dans tous ceux qui regroupaient un nombre presque infini de territoires moyens ou minuscules; cela explique les différences énormes qui existaient entre l'activité des différentes diètes circulaires: certaines ne se sont guère réunies depuis le XVII siècle, d'autres siégeaient presque tous les ans, comme celle de Franconie, où l'on compte trois cent vingt-deux sessions de 1517 à 1791 . Le rôle des cercles comme exécuteurs des jugements des tribunaux de l'Empire et des lois impériales fut confirmé par l'ordonnance concernant l'exécution des décisions impériales (Reichsexekutionsordnung), en 1555.

L'activité judiciaire reposait aux Temps modernes essentiellement sur deux cours souveraines: la Chambre impériale (Reichskammergericht) et le Conseil aulique (Reichshofrat). Le tribunal de la Chambre impériale fut l'une des créations les plus remarquables de la diète de Worms de 1495. Il poursuivit la tradition du tribunal de la cour royale médiévale. Une ordonnance promulguée en 1555 en fixa définitivement la forme. Siégeant à Spire depuis 1527 , il se transféra à Wetzlar à partir de 1689/1693. En français, on l'appelait souvent par le lieu de sa résidence, soit la Chambre de Spire, soit la Chambre de Wetzlar.

11 Cf. Alexander BEGERT, Böhmen, die böhmische Kur und das Reich vom Hochmittelalter bis zum Ende des Alten Reiches. Studien zur Kurwürde und zur staatlichen Stellung Böhmens, Husum 2003 (Historische Studien, 475).

12 La meilleure référence pour l'histoire de l'Italie impériale (Reichsitalien) est à l'heure actuelle le manuel de Karl Otmar Freiherr von ARETIN, Das Alte Reich 1648-1806, 3 vol., Stuttgart 1993-1997.

13 Cf., par exemple, HaRTMann, Der Bayerische Reichskreis, p. 24; SiCKEN, Der Fränkische Reichskreis p. 72-73. 
La Chambre impériale passait à juste titre (et passe encore de nos jours) pour le véritable tribunal de l'Empire, faisant preuve à plusieurs égards de corporatisme territorial. Premièrement, les ordres assuraient l'entretien financier du tribunal; deuxièmement, leur commission de visitation était chargée de l'inspection de la chambre; troisièmement, les états présentaient la plupart de ses magistrats: selon la grille fixée en 1555, les territoires contrôlaient ainsi vingt postes (dont deux pour les pays héréditaires autrichiens) sur vingt-cinq, l'empereur nominant les cinq autres candidats; d'après la grille établie par la paix de Westphalie en 1648, le nombre des magistrats étant porté à cinquante-cinq, l'empereur pouvait nominer le iudex de la chambre (les Français traduisaient le plus souvent par »grand juge «), ses quatre praesides (de facto, vice-présidents, dont deux devaient obligatoirement être de la confession d'Augsbourg) ainsi que deux des cinquante assessores (assesseurs), tandis que les états catholiques et les ordres protestants pouvaient présenter respectivement vingt-quatre assesseurs, cette présentation se faisant à travers les cercles de l'Empire ${ }^{14}$. Cette quasi-parité confessionnelle établie en 1648 contribuait aussi à souligner le caractère du tribunal comme représentation des états de l'Empire.

Au contraire, le Conseil aulique de l'Empire apparaissait plutôt comme un tribunal monarchique, puisqu'il était perçu comme l'instrument de justice contrôlé exclusivement par l'empereur. D'abord, le Reichshofrat fut créé et organisé selon la volonté des empereurs: Maximilien Ier ayant ébauché ses statuts dès 1498, une ordonnance impériale, promulguée sans le concours des états en 1654, fixa son organigramme et son fonctionnement dans la période que nous étudions. Composé théoriquement de dix-huit membres (dont un président et un vice-président) ${ }^{15}$, qui étaient tous nommés et appointés par l'empereur, ce tribunal comprenait seulement un tiers de magistrats protestants. Enfin, comme cour de justice aulique au sens propre du terme, il siégeait à la cour de l'empereur, c'est-à-dire, à la période qui nous intéresse, en règle générale à Vienne. De plus, l'empereur se réservait la décision de certaines causes majeures ${ }^{16}$.

Entre ces deux tribunaux d'Empire, il y avait une concurrence, c'est-à-dire que, dans la plupart des causes ${ }^{17}$, les parties pouvaient choisir la cour de justice à la-

14 Cf. le traité d'Osnabrück article V § 53, dans l'édition latine établie par Antje OschmanN, Acta Pacis Westphalicae, série III, section B: Verhandlungsakten, vol. 1: Die Friedensverträge mit Frankreich und Schweden, t. 1: Urkunden, Münster 1998 (APW III B 1/1), p. 126127; voir aussi le schéma de répartition du nombre de nominations d'assesseurs par cercle protestant, dans le même traité article V § 57, ibid., p. 128.

15 Contrairement à la Chambre impériale, qui peinait à atteindre ses effectifs théoriques, le Conseil aulique avait tendance à les dépasser.

16 En latin, on parlait alors de resolutiones Caesareae (résolutions de l'empereur), qui décidaient ces affaires.

17 Cependant, le Conseil aulique avait un domaine réservé qui comprenait notamment les causes pénales des états immédiats (c'est-à-dire les ordres qui relevaient directement de l'Empire, alors que les ordres médiats étaient soumis à un État territorial), certaines affaires réputées impériales (ratione causae; voir ci-dessous) et, depuis le XVII ${ }^{e}$ siècle, les affaires impliquant l'Italie. Cf, entre autres, Wolfgang SELLERT, Ưber die Zuständigkeitsabgrenzung von Reichshofrat und Reichskammergericht, insbesondere in Strafsachen und Angelegen- 
quelle elles désiraient s'adresser; en revanche, l'autre tribunal ne pouvait pas se saisir d'une affaire une fois que la procédure avait été engagée. D'autres tribunaux impériaux à caractère régional viennent compléter cet organigramme de la juridiction impériale, telle la chambre de Rottweil, qui suscitait parfois la curiosité des auteurs français. Il ne faut pas non plus oublier le système d'arbitrage (Austräge ou »austrègues«) hérité du Moyen Âge; dès 1495, ces austrègues prirent la forme de commissions judiciaires ${ }^{18}$.

Les travaux qui ont été consacrés ces dernières années à la Chambre impériale ont montré la fécondité de son activité jusqu'au crépuscule du Saint-Empire ${ }^{19}$; des dizaines de milliers de dossiers judiciaires en rendent témoignage. Bien que le fonctionnement du Conseil aulique soit moins bien connu, puisque ses actes n'ont pas encore fait l'objet d'un dépouillement systématique aussi exhaustif qu'il a été fait pour la Chambre impériale, le nombre extraordinaire des procès atteste son fonctionnement impeccable.

Pour comprendre la justice de l'Empire à l'époque moderne, il ne faut pourtant pas oublier qu'elle était essentiellement territorialisée, et qu'elle n'intervenait en première instance que dans certains cas bien définis. En règle générale, les sujets devaient avoir recours aux tribunaux de leurs seigneurs territoriaux respectifs, dans les causes civiles; ces tribunaux territoriaux exerçaient aussi la justice criminelle, et pouvaient même condamner à mort. Certains seigneurs jouissaient d'un privilège d'exemption ou d'un privilège dit de non appellando, qui limitait en appel ou excluait même catégoriquement le recours de leurs sujets à la justice impériale, de sorte qu'ils décidaient en dernier ressort ${ }^{20}$. En outre, le droit commun interdisait aux sujets territoriaux d'en appeler directement à la justice impériale en matière criminelle ${ }^{21}$. Même si nous n'étudierons pas ces cours de justice particulières, auxquelles les Français à l'époque moderne ne s'intéressaient guère, il ne faut pas oublier cette dimension territoriale de la justice en Allemagne.

Or, dans la plupart des cas où la partie citée était immédiate, la justice impériale devait déjà intervenir en première instance (intervention ratione personae). Cela veut dire que la plupart des procès politiquement importants car impliquant des seigneurs territoriaux étaient de son ressort. En outre, quand l'intérêt impérial

heiten der freiwilligen Gerichtsbarkeit, Aalen 1965 (Untersuchungen zur deutschen Staatsund Rechtsgeschichte, nouv. sér., 4); ID. (dir.), Reichshofrat und Reichskammergericht: ein Konkurrenzverhältnis, Cologne 1999 (Quellen und Forschungen zur höchsten Gerichtsbarkeit im Alten Reich, 34).

18 Le célèbre juriste allemand de la deuxième moitié du XVIII'e siècle, Johann Stephan Pütter, critiqua à juste titre un auteur français qui, au milieu du XVIIIe siècle, avait confondu les austrègues de l'époque moderne avec ceux du Moyen Âge; cf. [Johann Stephan] PưTTER, Litteratur des Teutschen Staatsrechts, 3 vol., Göttingen 1776-1783, t. II, p. 79, à propos de [Pierre-François LE COQ DE VILLERAY DE RouËR], Traité historique et politique du droit public de l'Empire d'Allemagne, Paris 1748.

19 Cf., par exemple, Bernhard Diestelkamp (dir.), Das Reichskammergericht. Der Weg zu seiner Gründung und die ersten Jahrzehnte seines Wirkens (1451-1527), Cologne 2004.

20 Malgré cela, les sujets avaient le droit d'attaquer leur seigneur en première instance devant les tribunaux d'Empire.

${ }^{21}$ En revanche, la justice impériale pouvait être saisie pour se pourvoir en cassation. 
était en cause, les tribunaux d'Empire pouvaient aussi se saisir en première instance des affaires concernant des sujets médiats (intervention ratione causae); ces causes, plutôt rares, concernaient en particulier les fiefs et les privilèges impériaux. Cependant, les sujets et les ordres médiats étaient dans la plupart des cas exclus de la justice impériale en première instance; dans certains territoires, ils ne pouvaient même pas recourir aux cours de justice impériales en deuxième instance contre les tribunaux impériaux. L'activité et le bon fonctionnement des hautes juridictions témoignent de la vivacité du Saint Empire romain germanique de 1648 à sa fin.

À côté des iura reservata et des iura comitialia que l'empereur exerçait de sa propre autorité ou en commun avec les états, sa fonction ne lui acquit pas de vastes domaines; en effet, les possessions impériales en Allemagne et en Italie avaient été usurpées ou aliénées avant la fin du Moyen Âge, de sorte qu'il ne restait à l'empereur que de modestes profits tirés de ses droits régaliens ${ }^{22}$. Si le projet de lever un denier commun (Gemeiner Pfennig), instauré en 1495, avait définitivement échoué au milieu du XVIe siècle, les contributions que les ordres devaient acquitter depuis 1507 pour financer la Chambre impériale (Kammerzieler) restaient en vigueur jusqu'à la fin du Saint-Empire.

$\mathrm{Si}$, à l'exception de certains impôts résiduels auxquels étaient soumises par exemple quelques villes impériales, ces contributions à l'entretien de la Chambre impériale représentaient le seul impôt permanent à l'époque moderne, la fiscalité ne fonctionnait pas si mal que l'historiographie avait tendance à le croire avant les travaux de Winfried Schulze sur le financement des guerres menées par l'empereur contre les Turcs ${ }^{23}$. Les contributions occasionnelles mais considérables que la diète d'Empire accordait à l'empereur étaient en général des subsides votés à des fins militaires. L'unité de base de ces contributions était appelée Römermonat, terme que les Français traduisaient littéralement par »mois romains«. Il s'agit d'une contribution votée dès le Moyen Âge, à l'occasion du Römerzug que le roi des Romains devait accomplir pour se faire couronner par le pape à Rome ou en Italie. À partir de 1521, la référence exacte fut la contribution consentie à Charles Quint pour la solde mensuelle des troupes qui devaient l'accompagner lors de son voyage à Rome. La répartition des levées se faisait à travers les cercles; les quotes-parts de chaque membre de l'Empire avaient été fixées par la matricule de Worms (Wormser Reichsmatrikel) de 1521. Parfois, la diète votait des contributions importantes qui pouvaient aller jusqu'à plus de deux cents mois romains ${ }^{24}$. Bien que l'empereur ne fût ainsi pas complètement dépourvu des moyens de dé-

${ }^{22}$ Sur les finances impériales en général dans la seconde moitié du XVII siècle, voir Jean BÉRENGER, Finances et absolutisme autrichien dans la seconde moitié du XVIIe siècle, Paris 1975 (université de Paris IV Paris-Sorbonne, série Sorbonne 1, travaux du Centre de recherches sur la civilisation de l'Europe moderne, 17).

23 Cf. Winfried ScHulze, Reich und Türkengefahr im späten 16. Jahrhundert. Studien zu den politischen und gesellschaftlichen Auswirkungen einer äußeren Bedrohung, Munich 1978.

24240 mois romains furent consentis, en 1641, à Ferdinand III. 
fendre l'Empire, cette forme de fiscalité empêchait la constitution d'une armée impériale permanente et indépendante des États territoriaux.

Jean-François Noël a raison de souligner que, globalement, »toutes ces institutions d'Empire n'apparaissaient pas en soi sensiblement plus mauvaises, ni plus rudimentaires que celles d'autres États de l'Europe moderne « 25 .

\section{2. »Droit « et »Constitution «: le rôle crucial des traités de Westphalie}

À l'époque que nous étudions, les Allemands aussi bien que les Français ont souvent jugé que la paix de Westphalie était l'acte juridique majeur et même la véritable Constitution du Saint Empire romain germanique. Ils pouvaient se fonder sur la teneur de la paix même. En effet, l'article XVII du traité d'Osnabrück ${ }^{26}$ stipule que

pour plus grande force et sûreté de tous et chacun de ces Articles, cette présente Transaction sera désormais une Loi perpétuëlle [perpetua lex] et une Pragmatique Sanction de l'Empire [pragmatica Imperii sanctio], ainsi que les autres Loix et Constitutions fondamentales de l'Empire [leges et constitutiones fundamentales Imperii], laquelle sera nommément insérée dans le prochain Résultat de l'Empire [Imperii recessui], et même dans la Capitulation Impériale [capitulationi Caesareae $]^{27}$.

La paix de Westphalie était donc appelée, dès 1648 , à faire date dans l'histoire constitutionnelle du Saint-Empire. Si, à première vue, la teneur de l'article semble évidente, ses termes juridiques nécessitent quelques remarques. D'abord, il est question des "autres Loix et Constitutions fondamentales de l'Empire «28. Or, quelles étaient ces lois et constitutions fondamentales, dont le traité d'Osnabrück (et celui de Münster) devait désormais faire partie? Selon Hartung, le concept "Grundgesetz « n'apparaît en Allemagne que pendant la guerre de Trente Ans; le premier document officiel où on l'utilise est la capitulation de Ferdinand III, suivie de la paix de Westphalie ${ }^{29}$. D'après Dickmann, il s'agirait d'une traduction du terme français »loi fondamentale «30. Si le concept était nouveau, la chose était déjà ancienne, et bien qu'aucune liste canonique n'en fût jamais dressée, il n'était

25 NoËL, Saint-Empire, p. 89.

26 Traité de paix d'Osnabrück article XVII $\S 2$ = traité de paix de Münster $\S 112$.

27 Texte latin d'après OschmanN, APW III B 1/1, p. 33 et 156; texte français d'après SCHEID, Traité sistématique, vol. IV, p. 290-291. On peut constater que toutes les traductions françaises de cet article traduisent les termes importants de la même façon.

28 Voir la citation précédente.

${ }^{29}$ Fritz HARTUNG, Deutsche Verfassungsgeschichte vom 15. Jahrhundert bis zur Gegenwart, Suttgart ${ }^{6} 1950$, p. 36.

30 Fritz DickmanN, Der Westfälische Friede und die Reichsverfassung, dans: Forschungen und Studien zur Geschichte des Westfälischen Friedens. Vorträge bei dem Colloquium französischer und deutscher Historiker vom 28. April-30. April 1963 in Münster, Münster 1965 (Schriftenreihe der Vereinigung zur Erforschung der Neueren Geschichte e.V., 1), p. 5-32, ici p. 9. 
point contesté quelles lois de l'Empire devaient être considérées comme appartenant au catalogue des lois fondamentales ${ }^{31}$. La paix de Westphalie fait fréquemment référence aux plus importantes d'entre elles: la Bulle d'or, la paix de religion de 1555, les capitulations impériales, etc. Dickmann définit la nature de telles lois:

Si l'on veut caractériser l'essence de ces »lois fondamentales «, l'on devra dire qu'elles représentaient des interventions modifiant une vie constitutionnelle qui évoluait toujours essentiellement en fonction de la tradition, de l'usage et de la coutume. Elles ne créèrent aucun droit nouveau mais fixèrent des parties du droit constitutionnel en vigueur, les soustrayant ainsi à tout changement ultérieur ${ }^{32}$.

La délimitation des droits de l'empereur et de ceux des états était leur objet principal $^{33}$. La qualification de la paix de Westphalie comme »loi fondamentale« et »constitution «, ne voulait donc pas dire qu'il s'agissait de créer un nouveau droit, mais de fixer les rapports entre l'empereur et les ordres tels qu'ils existaient déjà de facto. Notons à ce propos que le traité d'Osnabrück emploie ce mot (»constitution«) au pluriel. En effet, au milieu du XVIIe siècle, »constitution « était encore un synonyme de »loi, ordonnance, règlement ${ }^{34}$; l'emploi plus abstrait, signifiant la »Constitution de l'État«, n'est attesté pour la première fois qu'à la fin des années 1670 , dans la »Politique « de Bossuet, et n'entrera dans l'usage commun qu'au milieu du XVIIIe siècle ${ }^{35}$. La paix de Westphalie n'était donc pas appelée à être la Constitution future de l'Allemagne, mais à faire partie des lois réglant sa vie constitutionnelle ${ }^{36}$.

Le qualificatif »pragmatique sanction« attribué à la paix de Westphalie mérite aussi une explication. Selon Moréri, on donne le nom de »pragmatique sanction«, dont la signification originale serait contestée parmi les savants, »aux ordonnances qui concernent les grandes affaires de l'état ou de l'église, ou du moins les affaires de quelques communautés «37. Ce mot servait avant tout à donner à la paix

31 Ibid.

32 Il s'agit ici de notre propre traduction; pour des raisons évidentes d'exactitude terminologique, nous en donnons la version originale en allemand: "Will man das Wesen dieser -Grundgesetze < kennzeichnen, so wird man sagen müssen, daß sie gestaltende Eingriffe in ein Verfassungsleben darstellten, das sich im großen und ganzen immer noch nach Herkommen, Brauch und Gewohnheit weiterbildete. Sie schufen kein neues Recht, sondern fixierten Teile des geltenden Verfassungsrechts, die damit weiterem Wandel entzogen wurden «, ibid. 33 Ibid., p. 10.

${ }^{34}$ Cf. Wolfgang SchmalE, article »Constitution, Constitutionnel «, dans: Rolf REICHARDT, Eberhard SCHMITT, Hans-Ulrich THAMER (dir.), Handbuch politisch-sozialer Grundbegriffe in Frankreich 1680-1820, vol. 1-(19/20), Munich 1985-(2000) (Ancien Régime, Aufklärung und Revolution, 10), cahier 12, p. 31-63, ici p. 32.

35 Ibid., p. 33-34.

36 Dans ce contexte, il est à noter que le mot »droit « lui-même n'apparaît que très rarement au singulier sous l'Ancien Régime; »le droit«, au singulier, signifie en règle générale le droit romain. Cf. Wolfgang ScHMALE, article »Droit «, dans: REICHARDT, SCHMITT, THAMER (dir.), Handbuch politisch-sozialer Grundbegriffe, cahier 12, p. 65-87, ici p. 66-67.

${ }^{37}$ Cf. Louis MORÉRI, Le Grand Dictionnaire historique. Nouvelle [= vingtième] édition revue, corrigée et augmentée, 10 vol., Paris 1759, sub voce (s.v.) "Pragmatique Sanction«, t. VIII, p. 538-540, citation p. 538. 
de Westphalie un caractère inébranlable. Cette désignation devait particulièrement frapper les Français, qui aux XVII' et XVIIJe siècles entendaient sous le nom de "pragmatique sanction « principalement la pragmatique sanction de Saint Louis de 1268 et surtout celle de Charles VII de $1438^{38}$, et associaient donc ce terme aux moments les plus décisifs de l'histoire de France.

\section{Les fondements de la Constitution de l'Empire: lois écrites et coutumes}

Bien qu'un rôle primordial revînt incontestablement à la paix de Westphalie, le Saint-Empire n'avait pas de loi constitutionnelle unique qui réglât toute la vie politique. Ce qu'on peut appeler la Constitution de l'Empire se composait d'un certain nombre de lois organiques écrites, nommées lois fondamentales, et de coutumes qui n'étaient pas fixées par écrit. Les lois fondamentales avaient une importance particulière.

Le terme de »loi fondamentale« a déjà été défini; il n'y a pas de catalogue canonique de ces lois ${ }^{39}$. L'historiographie retient surtout les lois suivantes comme lois fondamentales du Saint Empire romain germanique: la Bulle d'or (Goldene Bulle, 1356); la paix publique perpétuelle de l'Empire (Ewiger Reichslandfrieden, 1495); les capitulations impériales jurées par les empereurs et les rois des Romains depuis Charles Quint (Kaiserliche Wahlkapitulationen, à partir de 1519); le traité de Passau (Passauer Vertrag, 1552); la paix de religion d'Augsbourg (Augsburger Religionsfrieden, 1555); la paix de Westphalie (Westfälischer Frieden, 1648). Les autres traités de paix passés par l'Empire, après 1648 , sont aussi considérés comme des lois fondamentales par certains auteurs de l'époque moderne; cependant, ces traités ont eu une influence moindre sur la Constitution de l'Empire, abstraction faite de certains articles, comme la quatrième clause du traité de Ryswick.

Outre ces lois fondamentales, certaines lois-cadres revêtaient une importance particulière; notamment, le $\mathrm{XVI}^{\mathrm{e}}$ siècle fut une époque de législation féconde: l'ordre public, y compris de nombreux aspects fondamentaux de la vie sociale et économique, fut réglé par les ordonnances de police (Reichspolizeiordnungen), qui datent surtout du milieu du XVIe siècle (1530-1570); la »Constitutio criminalis Carolina $\ll^{40}$, promulguée par Charles Quint en 1532 , et qui fut par ailleurs

38 Cf. ibid.; Pierre RICHELET, Dictionnaire françois, 2 vol., Genève 1679-1680 (réimpression: Genève 1970), t. II, p. 203.

39 Autant que nous sachions, il n'y a pas eu de disputes majeures sur ce sujet à l'époque moderne, étant donné que ces lois fondamentales ne tiraient pas leur force juridique du fait d'être considérées comme telles, mais d'autres facteurs (ratifications par la diète de l'Empire, etc.).

40 Gustav Radbruch (éd.), Die Peinliche Gerichtsordnung Kaiser Karls V. von 1532 (Carolina), sixième édition revue et publiée sous la direction d'Arthur KAUFMANN, Stuttgart 1996 (Reclam Universal-Bibliothek, 2990) [ $\left.{ }^{1} 1975\right]$. 
traduite en français ${ }^{41}$, posa les fondements du droit pénal allemand qui resta en vigueur jusqu'au siècle des Lumières; de même, les ordonnances monétaires du début de la deuxième moitié du XVIe siècle (1559-1566) font foi de l'activité législative intense au niveau de l'Empire, qui n'abandonna point toutes les prérogatives aux États territoriaux.

\section{La jurisprudence allemande (Reichspublicistik) dans la deuxième moitié du XVII ${ }^{\mathrm{e}}$ siècle: continuités et changements}

Dans le cadre de ce chapitre introductif, il n'est guère possible de donner une idée, si approximative soit-elle, des origines de la science du droit public dans l'Empire depuis le XVIe siècle. Car, comme le signale Stolleis, il est déjà difficile de dater le commencement de l'origine du droit public en tant que discipline universitaire en Allemagne, puisqu'il n'y a de père fondateur ni de date ou de lieu de naissance ${ }^{42}$. Cependant, il semble bien qu'un changement décisif se soit produit vers 1600 :

La situation se caractérise au contraire, autour de 1600 , par le fait que des cours et des disputes sur le sujet apparaissent presque simultanément en différents lieux, sans lien visible les uns avec les autres. La métaphore discutable de la »naissance « se justifie cependant ici, car même les contemporains notèrent avec étonnement l'apparition soudaine de la discipline ${ }^{43}$.

Avant la paix de Westphalie, les universités jouèrent un rôle secondaire, même si certains princes d'Empire saisirent l'occasion qui se présentait de défendre leurs libertés, en créant des chaires de ius publicum. Mais l'aspect le plus important, avant 1648 , fut la discussion acharnée de la forma Imperii, la forme du gouvernement du Saint-Empire, dans les publications de juristes tels que Paurmeister, Besold, Reinking, Limnaeus, Chemnitz, etc. Certes, les universités n'y furent pas pour rien ${ }^{44}$; en particulier, Marbourg et Giessen se livrèrent des combats académiques au sujet de la question de la forma Imperii.

Ce problème passant au second plan après $1648^{45}$, on avait coutume de qualifier le gouvernement du Saint-Empire de mixte ou de monarchique, tempéré d'éléments aristocratiques. La thèse selon laquelle le Saint-Empire serait la qua-

41 Ce fut Vogel, grand juge des gardes suisses à Paris, mort en 1749, et ayant poursuivi l'ouvrage de l'Allemand Jean Heiss sur l'histoire de l'Empire, qui établit, au XVIII' siècle, une édition française du Code criminel de Charles Quint, destinée à l'usage des conseils de guerre des troupes suisses.

42 Cf. STOLLEIS, Histoire du droit public, p. 207-215, en particulier p. 207.

43 Ibid., p. 207-208.

44 Stolleis a pourtant raison de souligner que l'âge d'or des universités ne commença qu'à partir de la fondation de l'université de Halle, en 1692, la plupart des écrits remarquables sur le droit public provenant d'auteurs extérieurs aux facultés de droit avant la fin du XVII ${ }^{\mathrm{e}}$ siècle, cf. ibid., p. 354-355.

45 Cela est vrai uniquement pour les auteurs allemands; nous verrons qu'il n'en va point de même pour les auteurs français. 
trième monarchie prophétisée par Daniel trouvait encore quelques adeptes. En revanche, la sacralité du Saint-Empire n'était guère remise en question.

La théorie du droit public que Stolleis qualifie de "baroque « ${ }^{46}$ reposait sur la perception de la réalité constitutionnelle formée par les traités de 1648 , les capitulations impériales de 1653 et surtout de 1658, l'ordonnance concernant le Conseil aulique, promulguée en 1654, et le recès de l'Empire de 1654. Nous n'aborderons pas ici les questions de méthode ${ }^{47}$. Parmi les auteurs les plus importants de la deuxième moitié du XVIIe siècle, il faudra citer les noms de Conring, de Pufendorf et de Leibniz. Ces auteurs étaient aussi bien connus en France, surtout Pufendorf.

\section{Autorité impériale et pouvoir des états}

À l'époque moderne, le bon fonctionnement des institutions impériales exigeait la collaboration de l'empereur et des états de l'Empire; depuis la Réforme, la confrontation religieuse entre les états catholiques et les ordres protestants, entre les luthériens et les calvinistes ainsi qu'entre l'empereur et les protestants risquait d'entraver le travail de ces institutions. Si la paix de religion d'Augsbourg de 1555 et la paix de Westphalie de 1648, mettant fin au demier conflit qui pouvait apparaître comme une guerre de religion dans l'Empire, arrivaient dans une certaine mesure à désamorcer ces conflits confessionnels, cette confrontation ne fut jamais surmontée jusqu'à la fin de l'Empire. En outre, le fonctionnement des institutions impériales dépendait des conjonctures des rapports de force politiques qui existaient entre l'empereur et les ordres. En effet, ces rapports ne furent pas définitivement réglés par la paix de Westphalie ni ne l'avaient été par aucun règlement antérieur. En revanche, les traités de Westphalie, qui n'évoquent, à aucun moment, les droits de l'empereur, en codifiant un certain nombre de droits revenant aux états, contribuèrent à une stabilisation de ces rapports.

Depuis 1438, les empereurs étaient tous issus de la maison d'Autriche, à une seule exception près (1742-1745), et celle-ci put conserver la dignité impériale jusqu'en 1806. Pour cette raison, il n'est pas surprenant que les Français de l'Ancien Régime aient identifié l'empereur au chef de la maison d'Autriche. Or, les assises patrimoniales que cette appartenance lui procurait consolidaient son autorité face aux états de l'Empire et en Europe. Cela est vrai non seulement au temps de Charles Quint mais aussi sous les règnes de ses successeurs, puisque malgré la perte de la monarchie d'Espagne au profit de Philippe II et de la branche espagnole de la maison, les empereurs et la branche de Vienne conservaient les couronnes de Bohême et de Hongrie ainsi que leurs vastes possessions allemandes et autrichiennes.

À plusieurs moments de l'histoire du Saint-Empire, les empereurs, forts de leurs prérogatives constitutionnelles et de la puissance de leurs pays héréditaires,

46 Cf. STOLleis, Histoire du droit public, p. 343.

47 À ce sujet, on pourra consulter STOLLEIS, Histoire du droit public. 
semblaient capables de vaincre le particularisme territorial et de transformer l'Empire en monarchie absolue. Si des projets de cette nature, à tort ou à raison, sont surtout attribués à Charles Quint et à Ferdinand II, l'emprise sur l'Empire que gardait un empereur régnant après 1648 tel que Léopold Ier ne fut pas non plus négligeable ${ }^{48}$. De plus, l'expérience de la puissance de ces deux empereurs, de la première moitié du XVIe siècle et de la première moitié du XVIIe, avait si profondément marqué les esprits en France que le retour à une telle situation politique et constitutionnelle dans l'Empire ne fut pas complètement exclu par certains auteurs français jusqu'au milieu du XVIIIe siècle.

Il est vrai pourtant que les libertés des états de l'Empire et l'autonomie de ceuxci concernant le gouvernement intérieur de leurs territoires étaient profondément ancrées dans la Constitution du Saint-Empire, surtout depuis la codification du droit des États territoriaux par la paix de Westphalie. Toutefois, Johannes Burkhardt a raison de souligner que la condition juridique des états de l'Empire fixée en 1648 n'était pas équivalente à la souveraineté, démentant une légende contraire qui avait trouvé un certain nombre d'adeptes, non seulement en France, mais aussi en Allemagne, surtout à l'époque du nationalisme ${ }^{49}$. Après la Seconde Guerre mondiale, c'est notamment Randelzhofer qui se fit le champion de cette légende »souverainiste « ${ }^{50}$. Cependant, la conclusion de Stolleis est sans appel:

Il a été amplement démontré que la thèse inspirée du droit des gens selon laquelle l'Empire aurait disparu comme »État « en 1648 pour se survivre comme association d'États souverains n'était pas conforme à la réalité historique. Les contemporains, dont le témoignage doit guider l'historien, étaient unanimes à reconnaître que l'Empire restait un »État«, aussi imparfait fût-il ${ }^{51}$.

Nous pouvons aussi souscrire sans réserve à un autre aspect que Burkhardt tient à souligner, et qui remet en question la prétendue imperfection du système étatique

48 Sur cet empereur, on a maintenant la très belle biographie de Jean BERENGER, Léopold Ier (1640-1705). Fondateur de la puissance autrichienne, Paris 2004.

49 Johannes BURKHARDT, Der Westfälische Friede und die Legende der landesherrlichen Souveränität, dans: Jörg ENGELBRECHT, Stephan LAUX (dir.), Landes- und Rechtsgeschichte. Festschrift für Hansgeorg Molitor zum 65. Geburtstag, Bielefeld 2004 (Studien zur Regionalgeschichte, 18), p. 199-220. Par ailleurs, NoËL combat la même idée reçue en France, cf. ID., Saint-Empire, p. 101-102: »Contrairement à une idée répandue, la paix de Westphalie n'a pas modifié sensiblement les sstructures du Saint-Empire héritées du temps de la Reichsreform du XVIe siècle, et elle n'a nullement fait des Reichsstände des >souverains $<$, puisqu'elle les maintient sans conteste sous la souveraineté de l'Empire, dont on aura encore l'occasion de voir les formes très effectives de survivance «. Nous voudrions aussi souligner la pertinence de ces propos de Noël à l'égard de l'origine des structures du Saint-Empire telles qu'elles existaient en 1648. Certains historiens français ont affirmé que la paix de Westphalie a conservé les structures politiques médiévales en Allemagne; or, comme Noël le remarque, ces structures médiévales avaient déjà été bouleversées par la Reichsreform à la fin du XVe et dans la première moitié du XVIe siècle, de sorte que si la paix de Westphalie a un esprit conservateur, celui-ci protège les structures héritées de l'époque de la Reichsreform, non celles du Moyen Âge.

50 Albrecht Randelzhofer, Völkerrechtliche Aspekte des Heiligen Römischen Reiches nach 1648, Berlin 1967 (Schriften zum Völkerrecht, 1).

${ }^{51}$ StolleIS, Histoire du droit public, p. 339. 
impérial: contrairement aux États de l'Europe occidentale, la création de l'État moderne fut, dans l'Empire, un processus plus complexe qui n'impliquait pas seulement l'État fédéral ni exclusivement les États territoriaux mais s'opérait aux niveaux et de l'Empire et des territoires ${ }^{52}$. Les droits que les princes et les villes pouvaient exercer à l'intérieur de leurs territoires étaient séculiers et religieux. Depuis 1555 , les princes jouissant du ius reformandi (droit de réformer) avaient la possibilité de décider de l'appartenance confessionnelle de leurs territoires, avec quelques exceptions concernant notamment les principautés ecclésiastiques catholiques. D'abord réservée aux catholiques et aux luthériens, la paix religieuse fut étendue aux calvinistes par la paix de Westphalie. Si la même paix confirmait en principe ce ius reformandi, la fixation de la carte confessionnelle telle qu'elle

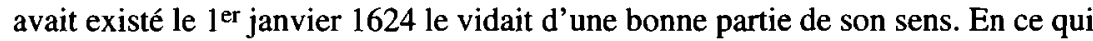
concerne les droits séculiers, outre la participation des états à la législation du Saint-Empire à travers la diète, ces traités consacrèrent de iure certaines prérogatives externes, comme le droit d'alliance et le droit de guerre (respectivement ius foederis et ius pacis ac belli) ainsi que l'autonomie interne des territoires, avec certaines restrictions imposées de la souveraineté de l'Empire. Bien que les ordres en eussent joui avant 1648 , cette codification ne fut pas sans importance, car elle les protégeait contre toute tentative ou velléité impériale de modifier en profondeur cette donne constitutionnelle. »Il était vain, désormais, de songer à un quelconque $>$ absolutisme $<$ impérial $\ll$, conclut Stolleis ${ }^{53}$.

\section{La diète permanente de Ratisbonne, pièce maîtresse du jeu diplomatique de la France en Allemagne}

La diète d'Empire, qui possédait l'essentiel du pouvoir législatif, s'était constituée en entité propre et différente des anciennes diètes de cour à partir de la fin du $\mathrm{XVe}$ siècle, de sorte qu'une partie de l'historiographie allemande plaide aujourd'hui pour limiter l'emploi du terme »diète d'Empire " (Reichstag) à la période postérieure à $1495^{54}$. Dans la mesure où l'Empire prit un caractère plus corporatif que monarchique, la formule »l'empereur et l'Empire« (Kaiser und Reich) changea de sens; d'abord complémentaire, le terme de Reich commença à dési-

52 Cf. Johannes BurkHardT, Das Reformationsjahrhundert. Deutsche Geschichte zwischen Medienrevolution und Institutionenbildung 1517-1617, Stuttgart 2002.

53 STOLLEIS, Histoire du droit public, p. 356.

${ }^{54}$ Le Reichstag a été l'objet de fructueuses recherches ces dernières années. Cf. notamment Helmut NeuHaus, Von Reichstag(en) zu Reichstag. Reichsständische Beratungsformen von der Mitte des 16. bis zur Mitte des 17. Jahrhunderts, dans: Heinz DuchHaRdT, Matthias SCHNETTGER (dir.), Reichsständische Libertät und habsburgisches Kaisertum, Mayence 1999 (Veröffentlichungen des Instituts für Europäische Geschichte Mainz, Abteilung Universalgeschichte, cahier suppl. 48), p. 135-149; Johannes HELMRATH, Der Weg zum Reichstag. Bemerkungen zu einer Neuerscheinung, dans: Zeitschrift für Historische Forschung 26 (1999), p. 61-74. 
gner une entité différente de l'empereur: les états d'Empire réunis à la diète 55 . Étant donné l'importance que les Français accordèrent au XVIIe siècle à la diète de l'Empire, sa composition et son fonctionnement doivent être évoqués ici.

À la diète, les seuls états immédiats (Reichsstände) étaient représentés, à l'exclusion de la chevalerie d'Empire (Reichsritterschaft), qui relevait pourtant directement de l'Empire, des ordres médiats et des sujets. S'il est vrai que la diète était essentiellement composée de villes et de princes allemands, l'Italie ne fut pas toujours complètement absente: cela explique aussi la présence, par exemple, d'un député du duc de Savoie au collège des princes d'Empire au congrès de Westphalie. La division de la diète en trois collèges (Reichtagskurien) - électeurs, princes et villes - reflétait la hiérarchie politique de l'Empire: le premier collège (Kurfürstenrat) était composé des princes électeurs à l'exclusion de celui de Bohême, qui le réintégra cependant en 1708; le deuxième collège (Fürstenrat) comprenait non seulement les autres princes séculiers et ecclésiastiques, mais aussi de simples comtes ou prélats ${ }^{56}$; le troisième collège (Städterat), regroupant les villes immédiates de l'Empire, au nombre d'une bonne cinquantaine au XVIIIe siècle, était divisé en deux bancs ${ }^{57}$ et ne jouissait de manière incontestable de son droit de vote décisif que depuis $1648^{58}$. Outre cette stratification des collèges, l'architecture de la diète était complétée par la division du collège des princes en banc laïque et en banc séculier - auxquels s'ajouta un banc oblique pour les principautés ecclésiastiques accordées aux protestants en 1648 - ainsi que par la constitution de deux corps à caractère confessionnel (Corpus catholicorum et Corpus evangelicorum) dans les affaires de religion, où la paix de 1648 avait prévu l'itio in partes des états.

La pérennisation de la diète à Ratisbonne, depuis 1663 , rompit avec la tradition des diètes temporaires et non périodiques, convoquées par l'empereur quand il avait besoin du concours des états, en règle générale dans une ville impériale du sud de l'Allemagne, proche de ses pays héréditaires ${ }^{59}$. Mais la diète ne devint perpétuelle qu'à cause de son incapacité de se terminer par un recès ordinaire; ce caractère ne découlait donc pas de la volonté des pouvoirs politiques d'instaurer un parlement permanent moderne. Il y a même des raisons concluantes pour voir

55 Pour l'histoire de la formule »l'empereur et l'Empire« (Kaiser und Reich), cf. Adalbert ERLER, article »Kaiser und Reich«, dans: ID., Ekkehard KAUFMANN, Dieter WERKMŬLLER (dir), Handwörterbuch zur deutschen Rechtsgeschichte, avec la collaboration philologique de Ruth SchmidT-WIEGAND, fondé avec le concours de Wolfgang STAMMLER, 5 vol., Berlin 1971-1996, vol. II (1978), col. 546-548. Erler souligne que cette formule pouvait signifier à la fois une unité et une dualité, et que depuis le XVII ${ }^{e}$ siècle, le concept »Empire« avait tendance à signifier en particulier les états d'Empire.

56 Ces derniers n'avaient pourtant pas de voix personnelles (»viriles «), comme les princes, mais les comtes votaient par »bancs« (Souabe, Franconie, Vetteravie, Westphalie) et les prélats par »curies« (Souabe, Rhénanie).

57 Souabe et Rhénanie.

58 En principe, ils avaient ce droit de vote depuis 1582 , mais ce n'est qu'avec les traités de Westphalie qu'il leur fut accordé expressément et incontestablement.

59 Ratisbonne avait été l'une des villes privilégiées dès le XVIe siècle: neuf diètes sur trenteneuf y avaient été organisées. 
dans la permanence de la diète le signe de son déclin: l'empereur fit sa dernière apparition à Ratisbonne en 1664; depuis, il s'y fit représenter par un principal commissaire et par un »concommissaire «. De même, les ordres envoyaient leurs députés à Ratisbonne au lieu d'y siéger en personne. Le directoire de la diète était une prérogative de l'archevêque de Mayence, archichancelier de l'Empire. Ses services étaient essentiels, car en faisant dicter les textes qui devaient servir de base aux délibérations de la diète (on parlait, à ce propos, de la »dictature de l'Empire«, Reichsdiktatur), tous les documents devaient passer entre ses mains.

La proposition de l'empereur (qu'on appelait décret de commission depuis que celui-ci était représenté par son principal commissaire) ayant été dictée et mise en discussion par les services de l'archichancellerie de Mayence, les trois collèges délibéraient séparément et votaient leur conclusum particulier. S'ensuivaient les négociations entre les deux premiers collèges (on disait alors "corrélation « en français à l'égard de cette procédure), qui aboutissaient au Conclusum duorum, c'est-à-dire à la conclusion des deux collèges supérieurs, qui était le fondement du Conclusum trium, de la conclusion des trois collèges, dès que les villes y adhéraient. Le résultat de ces négociations devait être approuvé par l'empereur pour obtenir valeur légale de conclusion d'Empire (Reichsschluss); sa promulgation, qui fut d'usage jusqu'à la permanence de la diète, en faisait un recès d'Empire (Reichsabschied).

À l'intérieur de chaque collège, on décidait à la majorité de voix, par »suffrage viril« ou collectif, pour les comtes, les prélats et les villes. Au sein de la diète, on se tenait en principe à la règle de l'unanimité. Mais dans les affaires de religion, les deux blocs confessionnels devaient trouver un accord à l'amiable (amicabilis compositio). En matière financière, certains états insistaient sur une restriction du vote majoritaire intracollégial au profit de l'unanimité. Au contraire, quand on ne pouvait aboutir à un accord entre les trois collèges, surtout lorsque les villes n'étaient pas de l'avis des deux collèges supérieurs, on passait souvent outre, en établissant un avis de l'Empire, qui expliquait les divergences que l'on n'avait pas su surmonter, la ratification par l'empereur servant d'arbitrage.

Les compétences essentielles de la diète étaient de trois sortes: légiférer, lever des impôts et déclarer la guerre ou faire la paix. Si l'empereur Ferdinand II avait encore insisté sur son droit d'interprétation authentique des lois impériales en promulguant l'Édit de restitution, en $1629^{60}$, la situation changea après les traités de Westphalie: l'ordonnance impériale du 16 mars 1654 concernant le Conseil aulique fut la dernière loi de l'Empire promulguée par un empereur (Ferdinand III) de sa propre autorité61. Très active au temps de la réforme des institutions impériales, à la fin du XVe et au XVIe siècle, la diète vécut un certain déclin au XVIIe siècle,

60 Cf. Michael FrISCH, Das Restitutionsedikt Kaiser Ferdinands II. vom 6. März 1629. Eine rechtsgeschichtliche Untersuchung, Tübingen 1993 (Jus ecclesiasticum. Beiträge zum evangelischen Kirchenrecht und zum Staatskirchenrecht, 44).

61 Cf. Wolfgang SelLeRT (éd.), Die Ordnungen des Reichshofrates 1550-1766, t. 2: [1626] bis 1766, Cologne 1990 (Quellen und Forschungen zur höchsten Gerichtsbarkeit im Alten Reich, 8/II), p. 1-260. 
malgré les compétences importantes qui lui furent accordées par les traités de Westphalie. Sur cet arrière-plan, il est compréhensible que les Français se soient désintéressés de cette institution après la fin du règne de Louis XIV. Passons donc de notre propre vision du Saint Empire romain germanique à l'Empire vu par les Français de l'Ancien Régime. 